\title{
Optimization of Total Variation regularization to improve the accuracy of the characterization of vertical cracks by lock-in vibrothermography
}

\author{
by A. Castelo*, A. Mendioroz* , R. Celorrio** and A. Salazar*
}

\begin{abstract}
* Departamento de Física Aplicada I, ETS Ingeniería, Universidad del País Vasco, UPV/EHU, Alda. Urquijo s/n, 48013 Bilbao, Spain, arantza.mendioroz@ehu.eus

**Departamento de Matemática Aplicada EINA/UIMA, Universidad de Zaragoza, Campus Río Ebro, Edificio Torres Quevedo, 50018 Zaragoza, Spain, celorrio@unizar.es
\end{abstract}

\begin{abstract}
We use lock-in vibrothermography to characterize vertical cracks. In the presence of ultrasounds, the rubbing of the crack faces produces heat which induces a temperature rise at the surface that is measured with an infrared camera. We fit data obtained by modulating the amplitude of the ultrasounds at several modulation frequencies. The inverse problem consisting of finding the geometry and location of the heat source from vibrothermography data is ill-posed, which makes it necessary to stabilize the inversion algorithm. We analyze the effect of the stabilizing term on the accuracy of the reconstructions.
\end{abstract}

\section{Introduction}

Ultrasound excited thermography is a very suitable technique for the detection of kissing cracks. The friction between crack lips in the presence of ultrasounds turns the crack into a heat source producing a temperature rise at the surface, on top of the crack. The goal of this work is to retrieve the dimensions and location of the heat source from lockin vibrothermography data. This inverse problem is ill-posed and as a consequence the minimization process is unstable. The algorithm can be stabilized by introducing a regularization term that is added to the residual to be minimized. In this work we analyze the effect of the regularization term and of the quality of the data on the quality of the reconstruction. To this purpose, we first calculate the surface temperature corresponding to a modulated vertical heat source. Then we invert synthetic data with added white noise by implementing two different regularization terms based on isotropic and anisotropic Total Variation functionals and we also check the effect of normalizing input data. Finally, we invert experimental data obtained on samples containing calibrated heat sources. The results show that it is possible to characterize $1 \mathrm{~mm}$ side square vertical cracks down to depths of about $3 \mathrm{~mm}$.

\section{Inverse problem and inversions of synthetic data}

The surface temperature $(z=0)$ is calculated by adding the contribution of point like modulated heat sources (at frequency $f, \omega=2 \pi f$ ) contained in a plane perpendicular to the sample surface at positions $\vec{r}_{0}$, covering the desired area $\Omega$. The sample is assumed to be semi-infinite and no heat losses are considered at the sample surface. Under these conditions, the temperature at the surface can be obtained as [1]:

$$
T\left(\vec{r}_{z=0}\right)=\iint_{\Omega} \frac{Q}{4 \pi K} \frac{e^{-q\left|\vec{r}-\vec{r}_{0}\right|}}{\left|\vec{r}-\vec{r}_{0}\right|} d \vec{r}_{0}^{3}
$$

where $q=\sqrt{2 \pi \text { if } / D}$ is the thermal wave vector, being $D$ the thermal diffusivity, $K$ is the thermal conductivity of the sample and $Q$ is the maximum heat flux delivered by the heat source. We generate surface temperature amplitude and phase data at nine at 9 modulation frequencies that we use in the experiments, namely, $0.05,0.1,0.2,0.4,0.8,1.6,3.2$, 6.4 , and $12.8 \mathrm{~Hz}$.

For the inversion, we integrate over the whole plane $\Pi$ containing the heat sources and we introduce a position and frequency dependent function $Q_{f}(\vec{r})=I_{f} Q(\vec{r})$ describing the heat source distribution as the product of a factor depending only on the modulation frequency $l_{f}$ and a normalized heat source distribution $Q(\vec{r})$. We want to calculate the heat source distribution that minimizes the square differences between the data and the calculated temperatures summed over all modulation frequencies. However, since the inverse problem is ill-posed, the minimization algorithm is unstable. We stabilize it by introducing a regularization term based on total variation (TV) functional multiplied by a regularization parameter $\alpha$. We make this choice because TV favors the search among blocky functions which helps determining the area occupied by the defect:

$$
R=\alpha T V(Q(\vec{r}))+\sum_{k=1}^{N}\left\|T_{\text {calc }}\left(I_{f_{k}}, Q(\vec{r})\right)-T_{f_{k}}\right\|^{2}
$$


Total variation has been implemented as the isotropic and anisotropic models:

$$
\begin{aligned}
& T V_{\text {ISOT }}(Q)=\iint_{S}|\nabla Q| d s \approx \lim _{i \rightarrow \infty} \iint_{S} \frac{\left|\nabla g_{i}\right|^{2}}{\sqrt{\varepsilon+\left|\nabla g_{i-1}\right|^{2}}} d s=\lim _{i \rightarrow \infty} \iint_{S} \frac{\left(\partial_{y} g_{i}\right)^{2}+\left(\partial_{z} g_{i}\right)^{2}}{\sqrt{\varepsilon+\left(\partial_{y} g_{i-1}\right)^{2}+\left(\partial_{z} g_{i-1}\right)^{2}}} d s \\
& T V_{\text {ANISOT }}(Q)=\iint_{S}\left(\left|\nabla_{x} Q\right|+\left|\nabla_{y} Q\right|\right) d s \approx \lim _{i \rightarrow \infty} \iint_{S} \frac{\left(\partial_{y} g_{i}\right)^{2}}{\sqrt{\varepsilon+\left(\partial_{y} g_{i-1}\right)^{2}}} d s+\lim _{i \rightarrow \infty} \iint_{S} \frac{\left(\partial_{z} g_{i}\right)^{2}}{\sqrt{\varepsilon+\left(\partial_{z} g_{i-1}\right)^{2}}} d s
\end{aligned}
$$

We have looked at the effect of these two models of the regularization term by inverting data corresponding to 1 $\mathrm{mm}$ side squares located at increasing depths with $5 \%$ added white noise and we have also compared the results when introducing either normalized or raw data into the algorithm. Figure 1 is a black and white representation of the normalized heat source distribution in the search plane. The real contours of the heat sources are depicted in red.

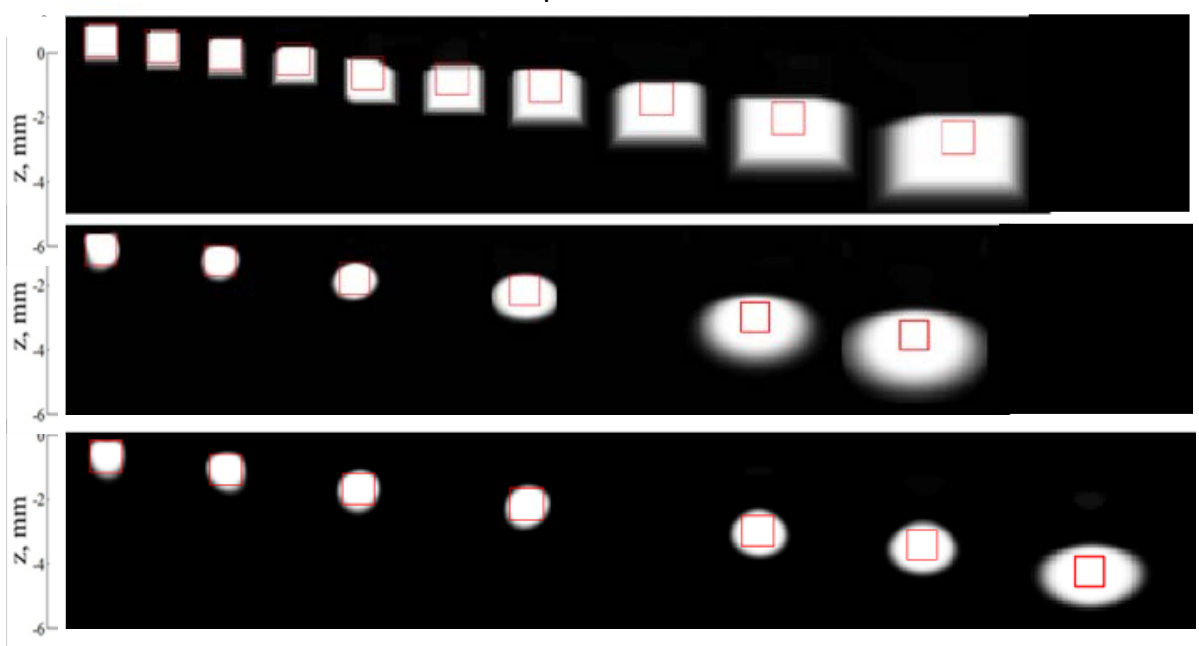

Fig. 1. Black and white representation of the normalized heat source distribution corresponding to synthetic data of square heat sources with 5\% added white noise retrieved by using normalized amplitudes and stabilizing the algorithm with the anisotropic TV model (top), with the isotropic model (center) and by using raw data and the isotropic model (bottom). Real contours are depicted in red.

As can be observed in figure 1, the effect of separating the derivatives in the two directions of the search plane is to favour jumps along the directions of the axes whereas in the isotropic model, the jumps occur in any direction, giving rise to round shapes. Moreover, entering raw instead of normalized data into the algorithm, further improves the quality of the reconstructions.

\section{Experiments}

We have prepared samples containing calibrated heat sources by attaching two steel parts with a flat common surface and putting $38 \mu \mathrm{m}$ thick cooper slabs of known dimensions in-between. When we launch the ultrasounds there is friction between the cooper slabs and the steel surfaces. We take data at a set of nine modulation frequencies mentioned above. In Fig. 2 we show reconstructions of experimental data obtained with $1 \mathrm{~mm}$ side square Cu slabs located at different depths introducing raw data in the algorithm and using the isotropic TV model. The reconstructions are very good for squares located down to $2.5 \mathrm{~mm}$ below the sample surface.

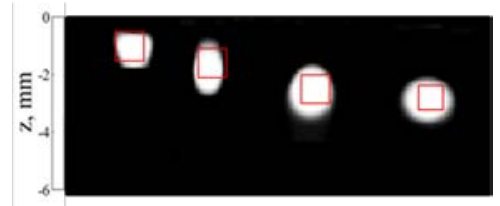

Fig. 2. Black and white representation of the normalized heat source distribution retrieved from experimental data obtained for different depths of a $1 \times 1 \mathrm{~mm}$ square Cu slab. Real contours in red.

This work has been supported by the Ministerio de Ciencia e Innovación (MAT2011-23811), by Gobierno Vasco (IT61913), by UPV/EHU (UFI 11/55), and by Diputación General de Aragón.

\section{REFERENCES}

[1] Mendioroz A., Castelo A., Celorrio R. and A. Salazar A., Characterization of vertical buried defects using lock-in vibrothermography. I. Direct problem. Meas. Sci. Tech. - Vol. 24 (2013) pp. 065601-06501/11 